\title{
Profile of Platelet Parameters in Coronavirus Disease - Positive Cases - An Observational Study
}

\author{
Nikhil Chaudhary ${ }^{1}$, Kalyani Raju ${ }^{1}{ }^{*}$,, Prabhakar Kamarthi ${ }^{2}$
}

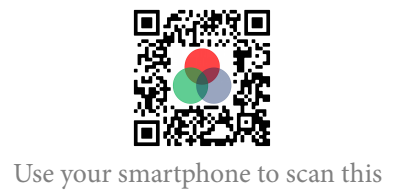

QR code and download this article

${ }^{1}$ Department of Pathology, Sri Devaraj Urs Medical College, Sri Devaraj Urs Academy of Higher Education and Research, Kolar, Karnataka, India

${ }^{2}$ Department of General Medicine, Sri Devaraj Urs Medical College, Sri Devaraj Urs Academy of Higher Education and Research, Kolar, Karnataka, India

\section{Correspondence}

Kalyani Raju, Department of Pathology, Sri Devaraj Urs Medical College, Sri Devaraj Urs Academy of Higher Education and Research, Kolar, Karnataka, India

Email: drkalyanir@rediffmail.com

History

- Received: Sep 28, 2021

- Accepted: Oct 23, 2021

- Published: Oct 29, 2021

DOI : 10.15419/bmrat.v8i10.700

\section{Check for updates}

\section{Copyright}

(c) Biomedpress. This is an openaccess article distributed under the terms of the Creative Commons Attribution 4.0 International license.

\begin{abstract}
Background: Coronavirus disease affects mainly the respiratory system. Other systems, including blood, are also affected. Blood cell abnormalities have varied between studies. The majority of patients present with platelet abnormalities. Methods: This was a laboratory observation study. All cases positive for the coronavirus disease 2019 (COVID-19) by reverse transcriptase - polymerase chain reaction (RT-PCR) test during the study period were considered for inclusion. Platelet index data were captured from an automated hematology analyzer: platelet count, mean platelet volume (MPV), platelet distribution width (PDW), plateletcrit (PCT), and platelet-large cell ratio (P-LCR). Platelet lymphocyte ratio (PLR), platelet neutrophil ratio (PNR), and platelet monocyte ratio (PMR) were calculated. The cases were classified into two groups: moderate and severe. The difference in alteration of platelet parameters between moderate and severe COVID-19 cases was analyzed using SPSS 22 version software. A p-value of $<0.05$ was considered statistically significant. Results Most cases (44.9\%) were in the age group of $41-60$ years. The male-to-female ratio was 1.9:1. Moderate cases comprised $53.4 \%$, and $46.6 \%$ of cases were severe. The association of PLR and PNR between moderate and severe cases was statistically significant. PLR was higher in severe cases than moderate cases, whereas PNR was higher in moderate cases than severe cases. Conclusions Studying platelet index profiles in COVID-19 patients can improve our limited knowledge of the disease progression regarding platelet parameters. PLR and PNR are the more reliable platelet parameters in managing COVID-19 patients, which help predict the prognosis and aid in improving therapeutic options for severe cases.
\end{abstract}

Key words: COVID-19, platelet lymphocyte ratio, platelet neutrophil ratio, platelet parameters

\section{INTRODUCTION}

Coronavirus disease affects mainly the respiratory system, but other systems can also be affected, including hematologic parameters. Blood cell abnormalities have varied between studies; most cases have shown decreased lymphocytes and neutrophilia, and a few have shown thrombocytopenia ${ }^{\mathbf{1}}$. The mechanisms by which coronavirus affects the hematopoietic system are not clear.

The majority of coronavirus infection patients present with platelet abnormalities. A possible mechanism may be a decrease in platelet production due to bone marrow aplasia, because of either the effect of cytokines or the direct effect of coronavirus on the bone marrow ${ }^{2}$.

This study reviews the changed platelet indices in coronavirus disease 2019 (COVID-19) disease. Interpreting these changes in patients infected with severe acute respiratory syndrome coronavirus-2 (SARS$\mathrm{CoV}-2$ ) could contribute to timely diagnosis and predict the prognosis.

\section{METHODS}

This laboratory-based observational study was performed over six months, from October 2020 to March 2021, at the Department of Pathology in a tertiary healthcare center in South India. The study obtained ethical clearance from the institute ethics committee. The objectives were to assess the profile of platelet parameters in COVID-19-positive cases and analyze the difference in the profile of platelet parameters between moderate and severe COVID-19-positive cases. All cases testing positive for COVID-19 by reverse transcriptase-polymerase chain reaction (RT-PCR) test were included. Cases with incomplete clinical details were excluded.

The sociodemographic data of all the cases were captured from the hospital record section. The platelet index data were collected from the hematology section at the Department of Pathology. The platelet parameters captured by automated hematology analyzer were platelet count, mean platelet volume (MPV), platelet distribution width (PDW), plateletcrit (PCT), and platelet-large cell ratio (P-LCR). Platelet lymphocyte ratio (PLR), platelet neutrophil ratio (PNR), and 
platelet monocyte ratio (PMR) were calculated using the platelet count, absolute lymphocyte count, absolute neutrophil count, and absolute monocyte count obtained with the automated hematology analyzer. The cases were classified into two groups: moderate and severe. Patients with $\geq 90 \%$ oxygen saturation were defined as moderate COVID-19, and patients with room air oxygen saturation $<90 \%$ were defined as severe COVID-19 $9^{3,4}$. The changes in platelet indices were analyzed for all cases. The difference in alteration of platelet parameters was analyzed between the moderate and severe COVID-19 groups.

The data were entered into a Microsoft Excel datasheet and analyzed using SPSS version 22 software (IBM SPSS Statistics, Somers, NY, USA). Categorical data were represented as frequencies and proportions. Continuous data were represented as mean and standard deviation. An independent t-test was used as a test of significance to identify the mean difference between two quantitative variables. A p-value of $<0.05$ was considered statistically significant after assuming all the rules of statistical tests.

\section{RESULTS}

A total of 410 cases were analyzed. Most cases (44.9\%) were in the age group of $41-60$ years (Table 1). Most were men, constituting 269 cases (65.6\%), and women represented 141 cases (34.4\%). The male-to-female ratio was 1.9:1 (Table 2). Moderate cases comprised $53.4 \%$, and $46.6 \%$ of cases were severe (Table 3 ).

The mean PDW was $12.09 \pm 2.67 \%$. Among moderate cases, the mean PDW was $11.96 \pm 2.68 \%$, and it was $12.25 \pm 2.66 \%$ in severe cases. The difference in PDW between moderate and severe cases was not statistically significant ( $p=0.272$; Tables 4 and 5).

The mean MPV was $10.48 \pm 1.08 \mathrm{fL}$. Among moderate and severe cases, the mean MPV was $10.4 \pm 1.13$ and $10.51 \pm 1.02 \mathrm{fL}$, respectively. The difference in MPV between moderate and severe cases was not statistically significant ( $\mathrm{p}=0.606$; Tables 4 and 5).

The mean P-LCR was $28.35 \pm 8.13 \%$. Among moderate cases, the mean P-LCR was $28.07 \pm 8.29 \%$, and it was $28.68 \pm 7.96 \%$ among severe cases. The difference in P-LCR between moderate and severe cases was not statistically significant ( $\mathrm{p}=0.448$; Tables 4 and 5).

The mean PCT was $0.34 \pm 1.56 \%$. Among moderate cases, the mean PCT was $0.43 \pm 2.13 \%$, and among severe cases, it was $0.24 \pm 0.09 \%$. The difference of PCT between moderate and severe cases was not statistically significant (p-value 0.222) (Tables 4 and 5). The mean PLR was $219.60 \pm 176.23 \%$. Among moderate cases, the mean PLR was $159.1 \pm 113.7 \%$, and among severe cases, it was $288.9 \pm 207.2 \%$. The difference in PLR between moderate and severe cases was statistically significant ( $p<0.001$; Tables 4 and 5). The mean PMR was $502.86 \pm 435.92 \%$. Among moderate cases, the mean PMR was $468.9 \pm 352.9 \%$, and it was $541.7 \pm 513.1 \%$ among severe cases. The difference in PMR between moderate and severe cases was not statistically significant ( $\mathrm{p}=0.091$; Tables 4 and 5). The mean PNR was $43.36 \pm 33.82 \%$. Among moderate cases, the mean PNR was $56.50 \pm 37.59 \%$, and among severe cases, it was $28.3 \pm 20.3 \%$. The difference in PLR between moderate and severe cases was statistically significant ( $\mathrm{p}<0.001$; Tables 4 and 5). The mean platelet count was $241.48 \pm 101.40 \times 10^{9} / \mathrm{L}$. Among moderate cases, the mean platelet count was $244 \pm 100 \times 10^{9} / \mathrm{L}$, and among severe cases, it was $239 \pm 103 \times 10^{9} / \mathrm{L}$. The difference in platelet count between moderate and severe cases was not statistically significant ( $\mathrm{p}=0.650$; Tables 4 and 5).

\section{DISCUSSION}

COVID-19 is a pandemic viral disease caused by the novel coronavirus SARS-CoV-2. Hematologic abnormalities are observed in COVID-19 patients. We aimed to analyze platelet parameters in moderate and severe COVID-19 cases.

In this study, male patients were predominantly affected, accounting for 269 cases (65.6\%); women constituted 141 cases $(35.4 \%)$. This was similar to the study by Ozcelik et al. ${ }^{3}$. In our study, most cases were in the age group of $41-60$ years $(n=184,44.9 \%)$. The cases were further subdivided into moderate and severe cases. More cases were categorized as moderate $(\mathrm{n}=219,53.4 \%)$.

PDW is a measurement of platelet anisocytosis, determined by calculating individual platelet volumes, and the normal range is $10.0-17.9 \%{ }^{5}$. In the present study, the mean PDW was within the normal range for both moderate and severe cases. Ozcelik et al. reported higher PDW levels in COVID-19-positive patients $^{3}$. In COVID-19, the PDW value is expected to be higher due to a cytokine storm leading to platelet production and destruction mechanisms. PDW determines the platelet size distribution range. A high PDW level indicates the destruction of immature platelets ${ }^{6}$. In our study, the normal PDW is probably due to a normal platelet count in the majority of cases (only $20 \%$ had thrombocytopenia).

MPV is a measure of the average size of platelets, and the normal range is $7.5-11.5 \mathrm{fL}^{3}$. The mean MPV was within the normal range for both categories of cases in the present study. MPV is considered an inflammatory marker, and it determines the size of 
Table 1: Distribution of subjects according to age group in the present study

\begin{tabular}{ccc}
\hline Age of subjects & Frequency & Percentage \\
\hline$<20$ yrs & 21 & $5.1 \%$ \\
$21-40 \mathrm{yrs}$ & 94 & $22.9 \%$ \\
$41-60 \mathrm{yrs}$ & 184 & $44.9 \%$ \\
$61-80 \mathrm{yrs}$ & 99 & $24.1 \%$ \\
$81-100 \mathrm{yrs}$ & 12 & $2.9 \%$ \\
Total & 410 & $100.0 \%$ \\
\hline
\end{tabular}

Table 2: Distribution of subjects according to gender in thepresent study

\begin{tabular}{ccc}
\hline Sex & Frequency & Percentage \\
\hline Female & 141 & $34.4 \%$ \\
Male & 269 & $65.6 \%$ \\
Total & 410 & $100 \%$ \\
\hline
\end{tabular}

Table 3: Distribution of subjects according to severity ofCOVID-19 disease

\begin{tabular}{ccc}
\hline COVID-19 & Subjects & Percentage \\
\hline Moderate & 219 & $53.4 \%$ \\
Severe & 191 & $46.6 \%$ \\
Total & 410 & 100.0 \\
\hline
\end{tabular}

Table 4: Profile of platelet parameters among cases in the present study

\begin{tabular}{lcccc}
\hline Platelet parameters & Minimum & Maximum & Mean & SD \\
\hline PDW (\%) & 0.1030 & 23.3000 & 12.095861 & 2.6740860 \\
MPV (fl) & 7.8000 & 17.2000 & 10.488049 & 1.0881575 \\
P-LCR (\%) & 8.2000 & 58.3000 & 28.357561 & 8.1372129 \\
PCT (\%) & 0.0200 & 30.0000 & 0.347805 & 1.5639965 \\
PLR & 10.8100 & 1304.3000 & 219.609537 & 176.2386736 \\
PMR & 30.7600 & 4081.8000 & 502.860683 & 435.9225552 \\
PNR & 0.9400 & 187.8000 & 43.369976 & 33.8220995 \\
Platelet count $\left(\mathrm{x} 10^{9} / \mathrm{L}\right)$ & 16 & 612 & 241.48 & 101.406 \\
\hline
\end{tabular}

PDW: Platelet Distribution Width, MPV: Mean Platelet Volume, P-LCR: Platelet- LargeCell Ratio, PCT: Plateletcrit, PLR: Platelet Lymphocyte ratio, PNR: Platelet Neutrophil ratio, PMR: Platelet Monocyte ratio 
Table 5: Comparison of profile of platelet parameters among moderate and severe cases of COVID-19

\begin{tabular}{lccccc}
\hline Platelet parameters & \multicolumn{2}{c}{ Moderate } & \multicolumn{2}{c}{ Severe } & p-value \\
& Mean & SD & Mean & SD & \\
\hline PDW (\%) & 11.9603 & 2.6827 & 12.2513 & 2.6627 & 0.272 \\
MPV (fl) & 10.4621 & 1.1384 & 10.5178 & 1.0298 & 0.606 \\
P-LCR (\%) & 28.0726 & 8.2944 & 28.6843 & 7.9623 & 0.448 \\
PCT (\%) & 0.4360 & 2.1365 & 0.2467 & 0.0956 & 0.222 \\
PLR & 159.1637 & 113.7943 & 288.9166 & 207.2759 & $<0.001$ \\
PMR & 468.911 & 352.9102 & 541.78 & 513.1374 & 0.091 \\
PNR & 56.5070 & 37.5945 & 28.3071 & 20.3429 & $<0.001$ \\
Platelet count (Thousands / cu.mm) & 244 & 100 & 239 & 103 & 0.650 \\
\hline
\end{tabular}

PDW: Platelet Distribution Width, MPV: Mean Platelet Volume, P-LCR: Platelet- Large Cell Ratio, PCT: Plateletcrit, PLR: Platelet Lymphocyte ratio, PNR: Platelet Neutrophil ratio, PMR: Platelet Monocyte ratio.

platelets ${ }^{7}$. MPV levels are increased in several diseases but reduced in viral diseases ${ }^{8,9}$. The reason for decreased MPV is the destruction of young platelets at the site of inflammation. Ozcelik et al. reported statistically significantly $(\mathrm{p}=0.027)$ decreased MPV in COVID-19 cases $^{3}$. Guçlu et al. reported a decrease in MPV at follow-up of COVID-19 patients, associated with significant mortality ${ }^{10}$. Our study did not include follow-up of cases.

P-LCR is defined as the percentage of platelets that surpass the normal platelet volume value of $12 \mathrm{fL}$ in the total platelet count. The P-LCR normal range is $15-35 \%{ }^{3}$. The mean P-LCR was slightly increased in both moderate and severe COVID-19 cases in the present study, but this was not statistically significant. P-LCR is a platelet index used to predict inflammation in numerous diseases, such as autoimmune diseases. Due to the expeditious inflammatory processes in COVID-19, severe patients have demonstrated increased P-LCR ${ }^{6,11}$.

PCT is a measure of total platelet mass, with a normal range of $0.20-0.36 \%{ }^{12}$. PCT in the present study was slightly higher in both moderate and severe COVID19 cases, but this was not statistically significant. He et al. found that PCT was not significant in COVID-19 cases $(\mathrm{p}=0.0545)^{11}$.

PLR is defined as the ratio of platelet to absolute lymphocyte counts. The normal range for PLR is $36.63-149.13 \%{ }^{13}$. The mean PLR was increased in severe COVID-19 cases compared to moderate cases, and this was statistically significant ( $\mathrm{p}<0.001)$. PLR has been advocated as a novel biomarker for predicting the prognosis and severity of COVID$19^{14}$. Simadibrata et al. found increased PLR in severe COVID-19 cases. The underlying pathogenesis of high PLR levels in severe COVID-19 cases is not clearly understood. A possible explanation is a decline in absolute lymphocyte count, which may be greater than the decline in platelet count, leading to increased PLR in severe cases ${ }^{15}$

PMR is defined as the ratio of platelet to absolute monocyte counts. The normal range is $7-22 \%$. In the current study, the mean PMR ratio was altered in both moderate and severe cases, indicating significant changes in monocytes and platelet counts, but the difference was not statistically significant between the groups. PMR parameters in COVID-19 cases have not been assessed by any study in the English literature to the best of our knowledge.

PNR is defined as the ratio of platelet to absolute neutrophil counts. The p-value was $<0.001$ between moderate and severe cases in the present study. PNR was more increased in the moderate COVID19 cases than severe cases. This could be due to a cytokine storm in severe cases of COVID-19, which causes recruitment of many neutrophils, neutrophilia, and increased absolute neutrophil count, resulting in decreased PNR. Hence, PNR may be a prognostic marker for COVID-19 cases. PNR has also been used as a prognostic marker in acute ischemic stroke ${ }^{\mathbf{1 6}}$. However, no published literature has been found regarding PNR in COVID-19.

In this study, thrombocytopenia was observed in $20 \%$ of cases, the majority of which were severe. However, no statistically significant difference existed between moderate and severe cases in platelet count. Evidence suggests that platelet and platelet indices have a role in prothrombotic responses to viral infections ${ }^{17}$.Some 
studies have reported a relationship between thrombocytopenia and severity of COVID-19, describing the mechanisms as suppression of bone marrow due to COVID-19 infection resulting in a reduction in platelet production, platelet destruction because of increased immune and inflammatory responses, and platelet consumption due to microthrombus formation in the lungs and other organs ${ }^{18,19}$. Endothelial cell damage causes activation and aggregation of platelets, leading to thrombus formation and causing depletion of platelets and eventually megakaryocytes, ultimately resulting in reduced platelet production ${ }^{19}$. Platelets also express surface receptors that enable the entry and binding of numerous viruses ${ }^{20}$.

This study's limitations were that it was unicentric with a relatively small sample size, and the cases were not followed up. However, estimation of platelet parameters is cheap and widely available, and some parameters can differentiate between moderate and severe COVID-19 cases. COVID-19 is a novel infection, so it is essential to recognize the biomarkers that may help predict the severity of the disease and aid as a prognostic factor. Research with a larger population is required to validate the findings of our study so that the information can be used for better care of COVID-19 patients.

\section{CONCLUSIONS}

Studying platelet index profiles in COVID-19 patients can improve our limited knowledge of disease progression regarding platelet parameters, help predict prognosis, and possibly aid in improving therapeutic options for severe cases. PLR and PNR are the more reliable platelet parameters in managing COVID-19 patients.

\section{ABBREVIATIONS}

COVID-19: Corona virus disease 2019

MPV: Mean Platelet Volume

PCT: Plateletcrit

PDW: Platelet Distribution Width

P-LCR: Platelet- Large Cell Ratio

PLR: Platelet Lymphocyte ratio

PMR: Platelet Monocyte ratio

PNR: Platelet Neutrophil ratio

RT-PCR: Reverse transcriptase polymerase chain reaction

\section{ACKNOWLEDGMENTS}

None.

\section{AUTHOR'S CONTRIBUTIONS}

Nikhil Chaudhary: Data collection, review of literature, writing manuscript; Kalyani Raju: Concept, review of literature, manuscript editing, manuscript revision; Prabhakar Kamarthi: Data collection, manuscript editing

\section{FUNDING}

None.

\section{AVAILABILITY OF DATA AND MATERIALS}

Data and materials used and/or analyzed during the current study are available from the corresponding author on reasonable request.

\section{ETHICS APPROVAL AND CONSENT TO PARTICIPATE}

This study was conducted in accordance with the amended Declaration of Helsinki. The institutional review board approved the study, and all participants provided written informed consent.

\section{CONSENT FOR PUBLICATION}

Not applicable.

\section{COMPETING INTERESTS}

The authors declare that they have no competing interests.

\section{REFERENCES}

1. Chen N, Zhou M, Dong X, Qu J, Gong F, Han Y. Epidemiological and clinical characteristics of 99 cases of 2019 novel coronavirus pneumonia in Wuhan, China: a descriptive study. Lancet. 2020;395(10223):507-13. PMID: 32007143. Available from: 10.1016/S0140-6736(20)30211-7.

2. Chan JF, Yuan S, Kok KH, To KK, Chu H, Yang J. A familial cluster of pneumonia associated with the 2019 novel coronavirus indicating person-to-person transmission: a study of a family cluster. Lancet. 2020;395(10223):514-23. PMID: 31986261. Available from: 10.1016/S0140-6736(20)30154-9.

3. Ozcelik N, Ozyurt S, Kara BY, Gumus A, Sahin U. The value of the platelet count and platelet indices in differentiation of COVID-19 and influenza pneumonia. Journal of Medical Virology. 2021;93(4):2221-6. PMID: 33135801. Available from: $10.1002 / j m v .26645$.

4. Guan W, Ni Z, Hu Y, Liang W, Ou C, He J, et al. Clinical characteristics of 2019 novel coronavirus infection in China. N Engl J Med. 2020;382(18):1708-1720. PMID: 32109013. Available from: 10.1056/NEJMoa2002032.

5. Chang D, Lin M, Wei L, Xie L, Zhu G, Cruz CSD. Epidemiologic and clinical characteristics of novel coronavirus infections involving 13 patients outside Wuhan, China. Journal of the American Medical Association. 2020;323(11):1092-3. PMID: 32031568. Available from: 10.1001/jama.2020.1623.

6. Gao Y, Li Y, Yu X, Guo S, Ji X, Sun T. The impact of various platelet indices as prognostic markers of septic shock. PLoS One. 2014;9(8):e103761. PMID: 25118886. Available from: 10.1371/journal.pone.0103761.

7. Mete E, Akelma AZ, Cizmeci MN, Bozkaya D, Kanburoglu MK. Decreased mean platelet volume in children with acute rotavirus gastroenteritis. Platelets. 2014;25(1):51-4. PMID: 23402274. Available from: 10.3109/09537104.2013.764493. 
8. Wang X, Meng H, Xu L, Chen Z, Shi D, Lv D. Mean platelet volume as an inflammatory marker in patients with severe periodontitis. Platelets. 2015;26(1):67-71. PMID: 24499137. Available from: 10.3109/09537104.2013.875137.

9. Karagöz E, Ulçay A, Turhan V. Mean platelet volume and red blood cell distribution width in prognosis of chronic hepatitis B. Wiener Klinische Wochenschrift. 2014;126(7-8):250-1. PMID: 24442860. Available from: 10.1007/s00508-013-0492-2.

10. Guclu E, Kocayigit H, Okan HD, Erkorkmaz U, Yurumez Y, Yaylacı S, et al. Effect of COVID-19 on platelet count and its indices. Rev Assoc Med Bras. 2020;66(8):1122-7. PMID: 32935808. Available from: 10.1590/1806-9282.66.8.1122.

11. He J, Wei Y, Chen J, Chen F, Gao W, Lu X. Dynamic trajectory of platelet-related indicators and survival of severe COVID-19 patients. Critical Care (London, England). 2020;24(1):607-11. PMID: 33054834. Available from: 10.1186/s13054-020-03339$\mathrm{x}$.

12. Kadhem SJ, Raheem AH, Aljumaily HS, Shammari AA, Humairi AK, Baay A, et al. Platelets Profile Changes in Patients with COVID 19. Sys Rev Pharm. 2020;11:569-74.

13. Liu X, Zhang $R$, He G. Hematological findings in coronavirus disease 2019: indications of progression of disease. Annals of Hematology. 2020;99(7):1421-8. PMID: 32495027. Available from: 10.1007/s00277-020-04103-5.

14. Simadibrata DM, Pandhita BA, Ananta ME. Platelet to lymphocyte ratio; A novel biomarker to predict the severity of COVID19 patients: A systematic review and meta-analysis. The Journal of the Intensive Care Society. 2020;p. 1751143720969587.
Available from: $10.1177 / 1751143720969587$

15. Henry BM, de Oliveira MH, Benoit S, Plebani M, Lippi G. Hematologic, biochemical and immune biomarker abnormalities associated with severe illness and mortality in coronavirus disease 2019 (COVID-19): a meta-analysis. Clinical Chemistry and Laboratory Medicine. 2020;58(7):1021-8. PMID: 32286245. Available from: 10.1515/cclm-2020-0369.

16. Jin P, Li X, Chen J, Zhang Z, Hu W, Chen L. Platelet-toneutrophil ratio is a prognostic marker for 90 -days outcome in acute ischemic stroke. Journal of Clinical Neuroscience. 2019;63:110-5. PMID: 30737090. Available from: 10.1016/j. jocn.2019.01.028.

17. Hottz ED, Bozza FA, Bozza PT. Platelets in immune response to virus and immunopathology of viral infections. Frontiers in Medicine. 2018;5:121. PMID: 29761104. Available from: 10. 3389/fmed.2018.00121.

18. Xu P, Zhou Q, Xu J. Mechanism of thrombocytopenia in COVID19 patients. Annals of Hematology. 2020;99(6):1205-8. PMID: 32296910. Available from: 10.1007/s00277-020-04019-0.

19. Guo L, Rondina MT. The era of thromboinflammation: platelets are dynamic sensors and effector cells during infectious diseases. Frontiers in Immunology. 2019;10:2204. PMID: 31572400. Available from: 10.3389/fimmu.2019.02204.

20. Chabert A, Hamzeh-Cognasse H, Pozzetto B, Cognasse F, Schattner M, Gomez RM. Human platelets and their capacity of binding viruses: meaning and challenges? BMC Immunology. 2015;16(1):26. PMID: 25913718. Available from: $10.1186 / \mathrm{s} 12865-015-0092-1$. 
Ready to submit your manuscript? Choose Biomedpress and benefit from:

- Fast, convenient online submission

- Through peer-review by experienced researchers

- Rapid publication on acceptance

- Free of charge (without publication fees)

Learn more http://www.biomedpress.org/journals/
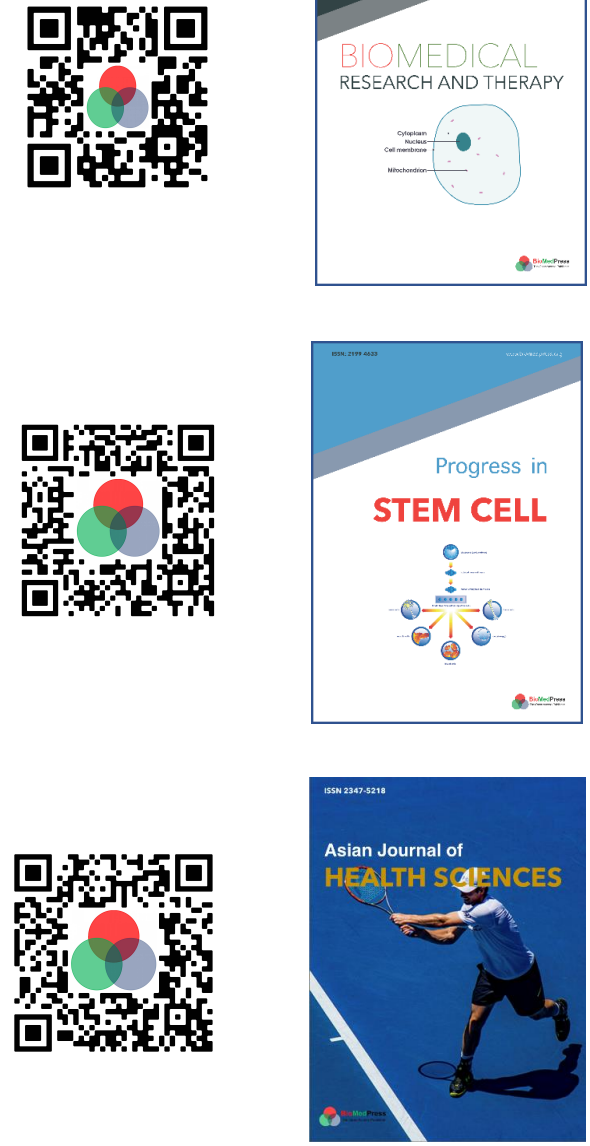

Asian Journal of Health Sciences

ISSN: 2347-5218

Indexed: Google Scholar

Acceptance Rate (2020): 72.89\%

Article Publishing Charge: Free

Submission to first editorial decision: 16.5 days

Biotechnological Research

ISSN: 2395-6763

Indexed: Google Scholar

Acceptance Rate (2020): $67.02 \%$

Article Publishing Charge: Free

Submission to first editorial decision: 28.5 days 